\title{
Perturbation study of the conductance through a finite Hubbard chain
}

\author{
Akira Oguri ${ }^{1}$ \\ Department of Material Science, Faculty of Science, Osaka City University, Osaka 558-8585, Japan
}

\begin{abstract}
The dc conductance through a Hubbard chain of size $N(=1,2,3, \ldots)$ connected to noninteracting leads is studied at $T=0$ in an electron-hole symmetric case using a perturbation theory in $U$. The result shows a typical even-odd property corresponding to a Kondo or Mott-Hubbard physics.
\end{abstract}

Keywords: quantum transport; electron correlation; Fermi liquid; mesoscopic system

Motivated by a current interest in effects of electron correlation on the transport through small systems, we have examined some theoretical approaches $[1,2]$. In this report, using a perturbation approach, we study the size $(N)$ dependence of the transport through a small interacting chain connected to semi-infinite leads.

We start with the Hamiltonian $\mathcal{H}=\mathcal{H}_{0}+\mathcal{H}_{I}$,

$$
\begin{aligned}
\mathcal{H}_{0}= & -\sum_{i=-\infty}^{+\infty} t_{i}\left(c_{i+1 \sigma}^{\dagger} c_{i \sigma}+c_{i \sigma}^{\dagger} c_{i+1 \sigma}\right) \\
& -\mu \sum_{i=-\infty}^{+\infty} n_{i \sigma}+\sum_{\substack{\sigma=1 \\
\sigma}}^{N}\left(\epsilon_{0}+\frac{U}{2}\right) n_{j \sigma}, \\
\mathcal{H}_{I}= & U \sum_{j=1}^{N}\left[n_{j \uparrow} n_{j \downarrow}-\frac{1}{2}\left(n_{j \uparrow}+n_{j \downarrow}\right)\right] .
\end{aligned}
$$

Here $c_{i \sigma}^{\dagger}$ is a creation operator for an electron with spin $\sigma$ at site $i, n_{i \sigma}=c_{i \sigma}^{\dagger} c_{i \sigma}$. The hopping matrix element is uniform $t_{i}=t$ except at the boundaries between the central region and two leads; $t_{0}=v_{L}$ and $t_{N}=v_{R}$.

At $T=0$, the dc conductance $g_{N}$ can be written in term of an inter-boundary element of a single-particle Green's function $G_{N 1}\left(\omega+i 0^{+}\right)$, and is determined by the value at the Fermi level $\omega=0$ as $g_{N}=\left(2 e^{2} / h\right) 4 \Gamma_{L}(0) \Gamma_{R}(0)\left|G_{N 1}\left(i 0^{+}\right)\right|^{2}$ [1]. Here $\Gamma_{\alpha}(0)=\pi D(0) v_{\alpha}^{2}$ with $\alpha=L, R$, and $D(0)=\sqrt{4 t^{2}-\mu^{2}} /\left(2 \pi t^{2}\right)$. The assumption made here is the validity of the perturbation theory in $U$. This seems to be probable for small $N$. In that case, the self-energy due to $\mathcal{H}_{I}$ has a property $\operatorname{Im} \Sigma_{j j^{\prime}}\left(i 0^{+}\right)=0$ at $T=0$ [3], and $g_{N}$ can be obtained through a scattering problem of free quasi-particles [2].

In this report, we consider an electron-hole symmetric case taking the parameters to be $\mu=0$ and $\epsilon_{0}+U / 2=0$. If the system has an additional in- 


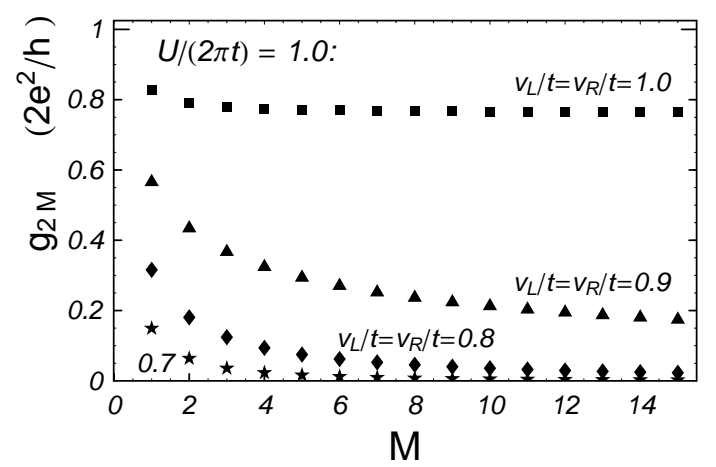

Fig. 1. $g_{N}$ vs $M$ for even $N(=2 M): U /(2 \pi t)=1.0$, and $v_{L} / t=v_{R} / t=0.7(\star), 0.8(\Downarrow), 0.9(\boldsymbol{\Delta}), 1.0(\mathbf{\square})$.

version symmetry $v_{L}=v_{R}$, it can be shown that a perfect transmission occurs $g_{N} \equiv 2 e^{2} / h$ for odd $N(=2 M+1)$ independent of the values of $U$ and $M$ [2]. This is caused by the Kondo resonance appearing at the Fermi level for odd $N$.

On the other hand, for even $N$, we evaluate the self-energy $\Sigma_{j j^{\prime}}\left(i 0^{+}\right)$within the second order in $U$ at $T=0$, and then obtain $G_{N 1}\left(i 0^{+}\right)$solving the Dyson equation in the real space [2]. In Fig. 1, $g_{N}$ for even $N(=2 M)$ is plotted vs $M$ for several values of $v_{L}\left(=v_{R}\right)$, where $U /(2 \pi t)=1.0$. The dc conductance decreases with increasing the size $2 M$. This behavior can be regarded as a tendency toward a Mott-Hubbard insulator, and it is pronounced for larger $U$. In Fig. 2, $g_{N}$ for even $N(=2,4, \ldots)$ is plotted vs $U$. The value of $g_{2 M}$ decreases with increasing $U$. When $v_{L}$ (or $v_{R}$ ) is smaller than $t$, the reduction of $g_{2 M}$ is proportional to $U^{2}$ at $U /(2 \pi t) \ll 1$. As it is seen in the plots for $v_{L}=v_{R}=0.7 t$ (dashed lines), the peak structure in the $U$ dependence becomes sharp for large $M$, and in the limit $M \rightarrow \infty$ the peak seems to vanish leaving the value at a singular point $U=0$ unchanged. In contrast, in the case of $v_{L}=v_{R}=t$ (solid lines), the reduction of $g_{2 M}$ is proportional to $U^{4}$ at $U /(2 \pi t) \ll 1$, and $g_{2 M}$ seems to be finite in the limit of large $M$. However, in order to verify this behavior for large $M$, the contributions of the higher-order terms should be examined because the unperturbed Hamiltonian $\mathcal{H}_{0}$ has a translational invariance accidentally in this case.

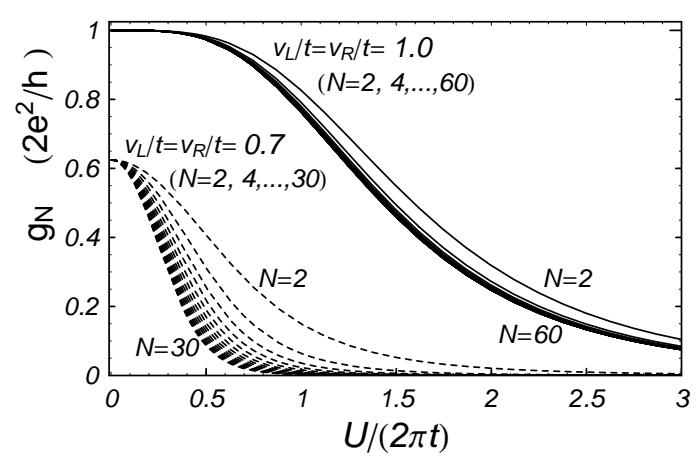

Fig. 2. $g_{N}$ vs $U$ for even $N(=2,4, \ldots): v_{L} / t=v_{R} / t=$ 0.7 (dashed lines), 1.0 (solid lines). $15+30$ lines are plotted.

Qualitatively, the even-odd property seems to be understood from that in the unperturbed system, especially from the level structure of the isolated chain. For odd $N$, there is a semi-occupied oneparticle state at the Fermi level $\omega=0$, and thus a doublet ground state is realized. When the leads are connected, the ground state is replaced by a Kondo singlet state and contributes to the tunneling. On the other hand, for even $N$, the Fermi level lies between the highest occupied level and the lowest unoccupied level, and thus a finite energy corresponding to the gap is necessary to excite the electrons. Although the levels are broadened by the coupling with the leads, the even-odd property is determined by whether there exists a zero-energy excitation or not. At finite temperatures, a characteristic energy scale of the Kondo or Mott-Hubbard physics will play an important role.

We expect that the model and approach used here can be applied to a series of quantum dots or a quantum wire of nanometer scale.

We thank H. Ishii for valuable discussions.

\section{References}

[1] A. Oguri, Phys. Rev. B 56, 13422 (1997); 58 1690(E) (1998).

[2] A. Oguri, Phys. Rev. B 59, 12240 (1999); unpublished.

[3] J. S. Langer and V. Ambegaokar, Phys. Rev. 121, 1090 (1961). 